\title{
Use of contraceptives, high risk births and under-five mortality in Sub Saharan Africa: evidence from Kenyan (2014) and Zimbabwean (2011) demographic health surveys
}

\author{
Admire Chikandiwa ${ }^{1 *}$ (D) Emma Burgess ${ }^{1}$, Kennedy Otwombe ${ }^{2}$ and Lucy Chimoyi ${ }^{3,4}$
}

\begin{abstract}
Background: Increasing uptake of modern contraception is done to alleviate maternal and infant mortality in poor countries. We describe prevalence of contraceptive use, high risk births, under-five mortality and their risk factors in Kenya and Zimbabwe.

Methods: This was a cross-sectional analysis on DHS data from Kenya (2014) and Zimbabwe (2011) for women aged 15-49. Geospatial mapping was used to compare the proportions of the following outcomes: current use of contraceptives, high-risk births, and under-5 mortality at regional levels after applying sample weights to account for disproportionate sampling and non-responses. Multivariate risk factors for the outcomes were evaluated by multilevel logistic regression and reported as adjusted odds ratios (aOR).

Results: A total of 40,250 (31,079 Kenya vs. 9171 Zimbabwe) women were included in this analysis. Majority were aged 18-30 years (47\%), married/cohabiting (61\%) and unemployed (60\%). Less than half were using contraceptives (36\% Kenya vs. $41 \%$ Zimbabwe). Spatial maps, especially in the Kenyan North-eastern region, showed an inverse correlation in the current use of contraceptives with high risk births and under-5 mortality. At individual level, women that had experienced high risk births were likely to have attained secondary education in both Kenya $(a O R=5.20$, 95\% Cl: 3.86-7.01) and Zimbabwe (aOR = 1.63, 95\% Cl: 1.08-2.25). In Kenya, high household wealth was associated with higher contraceptive use among both women who had high risk births (aOR: 1.72, 95\% Cl: 1.41-2.11) and under-5 mortality (aOR: 1.66, 95\% Cl: 1.27-2.16). Contraceptive use was protective against high risk births in Zimbabwe only (aOR: $0.79,95 \% \mathrm{Cl}: 0.68-0.92$ ) and under-five mortality in both Kenya (aOR: 0.79, 95\% Cl: 0.70-0.89) and Zimbabwe (aOR: $0.71,95 \% \mathrm{Cl}: 0.61-0.83$ ). Overall, community levels factors were not strong predictors of the three main outcomes.

Conclusions: There is a high unmet need of contraception services. Geospatial mapping might be useful to policy makers in identifying areas of greatest need. Increasing educational opportunities and economic empowerment for women could yield better health outcomes.
\end{abstract}

Keywords: Contraceptive use, High risk births, Kenya, Maternal mortality, Under-five mortality, Zimbabwe

\footnotetext{
* Correspondence: achikandiwa@gmail.com

${ }^{1}$ Wits RHI, Faculty of Health Sciences, University of the Witwatersrand, 22

Esselen St, Hillbrow, Johannesburg 2001, South Africa

Full list of author information is available at the end of the article
}

(c) The Author(s). 2018 Open Access This article is distributed under the terms of the Creative Commons Attribution 4.0 International License (http://creativecommons.org/licenses/by/4.0/), which permits unrestricted use, distribution, and reproduction in any medium, provided you give appropriate credit to the original author(s) and the source, provide a link to the Creative Commons license, and indicate if changes were made. The Creative Commons Public Domain Dedication waiver (http://creativecommons.org/publicdomain/zero/1.0/) applies to the data made available in this article, unless otherwise stated. 


\section{Background}

The third Sustainable Development Goal of the United Nations aims for healthy living and well-being for all and targets reduction of maternal mortality ratio (MMR) to under 70 deaths per 100,000 live births and under-5 mortality rate (U-5MR) to below 25 per 1000 live births respectively [1]. More effort will be required in developing regions and especially Sub-Saharan Africa [SSA] where MMR remains way higher than in developed regions [2] and eight of ten deaths in children is under the age of five [3].

Increasing access to contraceptive methods has been recommended for the reduction of MMR and U-5MR [4]. The impact of contraceptive use on mortality is mediated through factors that include early births (mother's age is below 18 years), giving birth late (mother's age is above 34 years), short period of time between births (less than 2 years), and high parity rates (i.e. $>3$ children) which are often referred to as high risk births as they represent increased chance of mortality to both the mother and child [5-7].

In SSA, the contraceptive prevalence rates have remained low, and the region continues to face high MMR and U-5MR [8]. The region is characterized by high fertility and often unwanted pregnancies, which could be due to low contraceptive usage [9]. Available data from Kenya and Zimbabwe has suggested that child mortality rates are related to maternal factors which include age at childbirth, spacing of births and parity $[10,11]$.

Prior research from SSA shows that contraceptive coverage is further influenced by a number factors including: medical barriers such as accessibility of the services, health care workers attitudes, medical and regulatory guidelines; [12] cultural norms such as the role of women in decision making, place of residence (i.e. rural vs urban), religious and cultural beliefs [13] as well as individual level factors which include age, education, employment, marital status, parity [14]. Community level factors which include, illiteracy rate and poverty, have been shown to influence uptake of healthcare services especially in rural areas [15]. There are also other factors that influence MMR and U-5MR beyond contraception like accessing education and quality of antenatal care $[16,17]$. However, there is scarcity of data exploring usage of contraceptives and outcomes such as high risk births as well as under-five mortality from geospatial distributions and contextual community factors using multi-country data. To address this gap, we conducted a comprehensive comparative cross-sectional analysis using the most recent Demographic Health Survey (DHS) data from Kenya (2014) and Zimbabwe (2011) at the time, to investigate the (i) spatial distribution of contraceptive coverage, prevalence of high-risk births and under-five mortality, and (ii) correlates of contraceptive usage at individual and community level among women experiencing under-five mortality or high risk births.

\section{Methods}

\section{Study design, population and sample size}

This population-based study used data from Kenya DHS 2014 and Zimbabwe DHS 2011 focussing on females in the child-bearing age of 15-49 years. The DHS has a two-stage cluster sampling technique using two strata; place of residence (rural/urban) or administrative regions. A sample of 1612 (Kenya) and 406 (Zimbabwe) clusters was drawn from previous census by the Kenya National Bureau of Statistics and the Zimbabwe National Statistics Agency respectively. Each cluster randomly selected from an enumerated area (EA) contained a total of 25 (Kenya) and 27 (Zimbabwe) EAs. We used individual weighted data on 31,079 and 9171 women and their associated child records from Kenya and Zimbabwe, respectively. During analysis, we used variables with up to $85 \%$ of their observations available. We also created composite variables that combined information from other variables as described in the next section.

\section{Data collection and variables}

Information on sampling techniques and procedures applied for data collection in both countries have been published in the final DHS reports $[18,19]$.

\section{Outcome variables}

Three outcomes were investigated in the analysis. Primarily, modern contraception use, defined as reporting the use of any modern contraception by women in a union aged 15-49. As per DHS definition, modern contraception included the following: female or male sterilisation, intrauterine contraceptive devices, hormonal methods such as oral contraceptive pill, injectables and implants, barrier methods like female or male condoms, diaphragms, as well as spemicides and lactational amenorrhoea. Among women who had ever reported a live birth in their lifetime we also assessed: under-five child mortality, defined as death of a child aged five years and below, and high-risk births, defined as giving birth below 18 years or above 35 years of age, of high birth order ( $>3$ children), or with short spacing $(<2$ year-gap between children). All these three outcomes were binary (i.e. yes which was coded as 1 or no coded as 0 ).

\section{Explanatory variables}

Individual and community characteristics were examined for possible associations with contraceptive use (in women that experienced high risk births under-five child mortality), high-risk births and under-five child mortality. Characteristics were chosen because previous studies have found them to be important factors [13, 15]. Individual variables included age of the woman, level of education (primary/secondary/beyond secondary), employment status (yes/no), marital status (never/previously/currently 
married), number of children and birth order, wealth index (high/middle/low) and religion. Community-level variables were calculated using the primary sampling unit (PSU) of the data. Community characteristics were selected to consider the socio-economic status of the community. This was done by combining four factors; place of residence (rural/urban), proportion of illiteracy, poverty and unemployment. Place of residence was a direct level independent variable whereas proportion of illiteracy, poverty and employment were aggregates of individual independent variables. The aggregates were proportions generated within PSUs by dividing the prevalence of each factor (i.e. those with any employment) in the PSU by the number of women in the PSU [20].

\section{Data analyses}

We analysed data using Stata 14.1 software [21] and choropleth map creation using ArcMap 10.4 [22]. The statistical methods used are adopted from similar work conducted in Burkina Faso by Maiga et al. [8].

\section{Descriptive analysis}

Descriptive statistics such as medians and interquartile ranges (IQR) for continuous measures were determined for participant and community characteristics. Sample weights were applied on the estimation of proportions and frequencies to adjust for disproportionality due to non-response. Cross tabulations were conducted on categorical variables to determine their association. Choropleth maps were used to display the geographical distribution of the outcome variables.

\section{Measures of association (fixed effects)}

Risk factors for high-risk births, usage of contraceptives among women experiencing high risk births and under-five mortality were assessed by multilevel logistic regression models. These were fitted under univariate and multivariate models at individual and community levels. Odds ratios and their 95\% confidence bands were used to assess the measure of association. Interpretations in the manuscript were based on the adjusted Odds ratios (aOR) from the multivariate models.

\section{Assessing variation between communities using the random effects model}

Variability within communities was evaluated using the random effects model. Additionally, variability changes in the empty and consecutive model were assessed proportionately using the proportional change in community variance $(\mathrm{PCV})[20]$.

\section{Modelling approaches}

The logistic regression model was fitted due to the binary nature of the dependent variable whereas the hierarchical nature of the DHS data allowed for the fitting of multilevel models in which individual women (level 1) were nested in their communities (level 2). This allowed for the modelling of relationships between independent variables on the dependent outcome. In order to decompose the total variance shared at the individual and community levels, an empty model was fitted. All the individual-level factors were included in model 2 and community-level factors into model 3 while the model 4 comprised individual-level and community-level factors.

\section{Model fitness and precision}

In the individual and community level multivariate regressions, the log-likelihood and Akaike Information Criterion (AIC) were used to assess how well the model fitted, taking the one with the smallest value as the best.

\section{Results \\ Descriptive analysis}

A total of 40,250 women were eligible for inclusion in this study; 31,079 from Kenya and 9171 from Zimbabwe. Majority were of age $18-30$ years ( $48 \%$ vs. $46 \%)$, married/living with a partner (61\% vs. $61 \%)$ and unemployed in the past 12 months (62\% vs. 59\%) in Kenya and Zimbabwe (Table 1). Less than half of the women reported current contraceptive use (36\% and $41 \%)$ where the main route of administration was injections (18\%) and oral (26.4\%) contraceptives in Kenya and Zimbabwe respectively. Majority of the women in Kenya had attained primary education (50\%) while Zimbabweans had at least attained secondary education (69\%). Distribution of contraception coverage, high-risk birth and under-five mortality from both countries are presented in Figs. 1, 2 and 3. In Kenya, low contraceptive coverage was reported in North-Eastern province (3\%); high under-five child mortality in Nyanza province (20\%); and more high-risk births in North-Eastern province (28\%). The North-Eastern province which had the lowest coverage for contraceptive (3\%), had a higher prevalence of under-five child mortality (14\%) and the highest prevalence of high-risk births. In Zimbabwe, there was low contraceptive usage in Matabeleland South (45\%); high under-five child mortality in Mashonaland Central (16\%) and Manicaland (16\%); and more highrisk births in Mashonaland Central (44\%). Mashonaland central had the highest contraceptive coverage (61\%) and also the highest prevalence of under-five child mortality (16\%) and high-risk births (44\%). Further comparisons in these countries by rural-urban divide are presented in Additional file 1: Figure S1, Additional file 2: Figure S2, Additional file 3: Figure S3, Additional file 4: Figure S4. 
Table 1 Baseline demographic characteristics of women who have ever given birth in the Kenyan $(N=31,079)$ and Zimbabwean $(N=9171)$ DHS

\begin{tabular}{lll}
\hline Characteristic & Kenya & Zimbabwe \\
& $\mathrm{N}(\%)$ & $\mathrm{N}(\%)$ \\
\hline
\end{tabular}

Individual level factors

Age categories

$<18$ years

18-30 years

$>30$ years

Marital status

Married/ living with partner

Divorced/ separated/widowed

Never in union

Employment in past 12 months?

Yes

No

Modern contraceptive use

Yes

No

Modern Contraceptive type

None

Oral

IUD

Injection

Condoms

Implants

Sterilization

Other

Mothers education level

None

Primary

Secondary or higher

Household wealth index

Low

Middle

High

Mothers religion

None

Roman Catholic

Protestant

Muslim

Other

Number of children

$$
0
$$

$\begin{array}{ll}3769(12.1) & 1603(17.5) \\ 14,921(48.0) & 4181(45.6) \\ 12,389(39.9) & 3387(36.9)\end{array}$

19,036 (61.3)

$5578(60.8)$

$3468(11.1)$

$8575(27.6)$

$680(7.4)$

2913 (31.8)

5628 (38.2)

$3776(41.2)$

$5395(58.8)$

$9111(61.8)$

11,025 (35.5)

3711 (40.5)

19,047 (61.3)

$5388(58.8)$

$19,047(61.3)$

$5388(58.8)$

$1439(4.6)$

$614(2.0)$

$5516(17.8)$

797 (2.6)

1969 (6.3)

655 (2.1)

1, 042 (3.4)

4183 (13.5)

$15,613(50.2)$

$11,283(36.3)$

13,232 (42.6)

3292 (35.9)

5946 (19.1)

11,901 (38.3)

1589 (17.3)

4290 (46.8)

506 (1.6)

6229 (20.0)

$20,072(64.6)$

4, 161 (13.4)

$11(0.4)$

7834 (25.2)

4426 (14.2)
Table 1 Baseline demographic characteristics of women who have ever given birth in the Kenyan $(N=31,079)$ and Zimbabwean ( $N=9171)$ DHS (Continued)

\begin{tabular}{|c|c|c|}
\hline \multirow[t]{2}{*}{ Characteristic } & Kenya & Zimbabwe \\
\hline & $\mathrm{N}(\%)$ & N (\%) \\
\hline $2-3$ & $8856(18.5)$ & $3042(33.2)$ \\
\hline$>3$ & $9963(32.1)$ & $1961(21.4)$ \\
\hline \multicolumn{3}{|c|}{ High risk births } \\
\hline No & $16,742(72.0)$ & $3729(55.5)$ \\
\hline Yes & $6503(28.0)$ & $2996(44.5)$ \\
\hline \multicolumn{3}{|c|}{ Under 5 child mortality in the last 5 years } \\
\hline No & $19,307(83.1)$ & $5745(85.4)$ \\
\hline Yes & $3938(16.9)$ & $980(14.6)$ \\
\hline \multicolumn{3}{|c|}{ Community level factors } \\
\hline \multicolumn{3}{|c|}{ Place of residence } \\
\hline Urban & $11,614(37.4)$ & 3437 (37.5) \\
\hline Rural & $19,465(62.6)$ & $5734(62.5)$ \\
\hline \multicolumn{3}{|c|}{ Proportion of unemployment* } \\
\hline $0 \%$ & $29,958(96.4)$ & $151(1.7)$ \\
\hline Up to $10 \%$ & $812(2.6)$ & $836(9.1)$ \\
\hline Up to $50 \%$ & $309(1.0)$ & $7,489,981.7)$ \\
\hline Above $50 \%$ & - & $695(7.6)$ \\
\hline \multicolumn{3}{|c|}{ Proportion of illiteracy } \\
\hline $0 \%$ & $3193(10.3)$ & $7972(57.0)$ \\
\hline Up to $10 \%$ & $6251(20.1)$ & $1185(12.9)$ \\
\hline Up to $50 \%$ & $16,595(53.4)$ & $4(0.1)$ \\
\hline Above $50 \%$ & $5040(16.2)$ & - \\
\hline \multicolumn{3}{|l|}{ Poverty level } \\
\hline $0 \%$ & 5778 (18.6) & $4290(46.8)$ \\
\hline Up to $10 \%$ & $2282(7.3)$ & $4,875(53.2)$ \\
\hline Up to $50 \%$ & $10,652(34.3)$ & $6(0.1)$ \\
\hline Above 50\% & $12,367(39.8)$ & - \\
\hline
\end{tabular}

*52.6\% missing information

\section{Multivariate analysis Individual level factors}

Table 2 presents risk factors for contraceptive usage in women stratified by high-risk birth and under-five mortality in both countries. At individual level in Kenya among women who have experienced high-risk births, there were higher odds for contraceptive use in those who were married or living with a partner (aOR: 2.98, 95\% CI: 1.67-5.29), had primary (aOR: 4.57, 95\% CI: $0.36-5.77)$ and secondary or higher level of education (aOR: 5.20, 95\% CI: 3.86-7.01). Those $>34$ years of age had lower odds (aOR: 0.57, 95\% CI: 0.48-0.68). In Zimbabwe, primary (aOR: 1.53, 95\% CI: 1.04-2.25) and secondary or higher level of education (aOR: 1.63, 


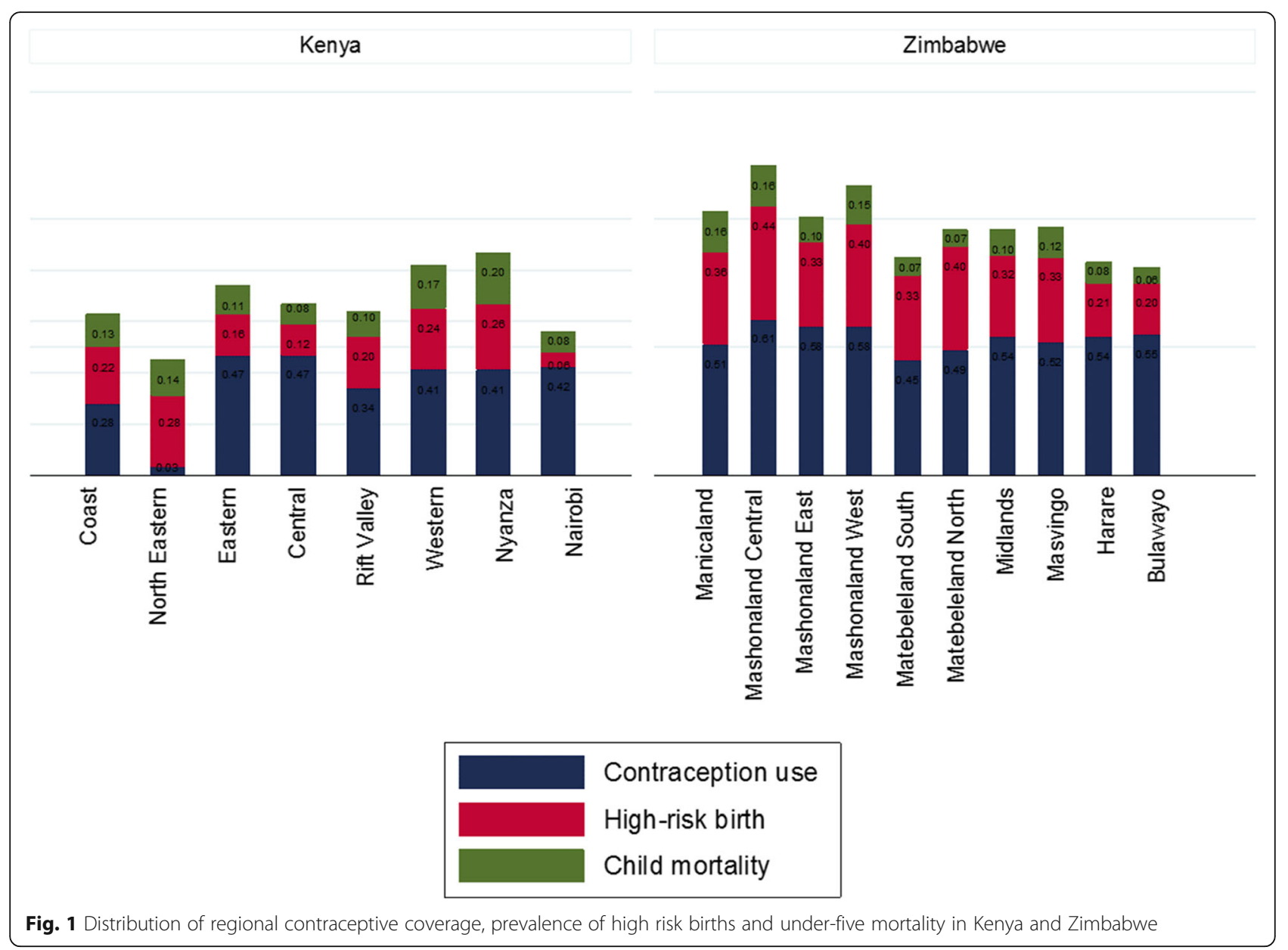

95\% CI: 1.08-2.47) and employment in the past 12 months (aOR: 1.27, 95\% CI: 1.07-1.51) was associated with higher odds for modern contraceptive use. Those married or living with partner (aOR: 0.29, 95\% CI: 0.21-0.40) and previously married (aOR: 0.19, 95\% CI: 0.14-0.26) experienced lower odds.

Married or living with a partner (aOR: $2.48,95 \% \mathrm{CI}$ : 1.37-4.50), primary (aOR: 4.9, 95\% CI: 3.48-6.90) and secondary or higher level of education (aOR: 4.14, 95\% CI: 2.73-6.28) was associated with higher odds for modern contraceptive use among Kenyan women who experienced under-five mortality. Age $>34$ years was associated with lower odds (aOR: 0.72, 95\% CI: 0.59-0.87). In Zimbabwe, married or living with partner (aOR: 0.30, 95\% CI: $0.17-0.51$ ) and previously married (aOR: $0.18,95 \% \mathrm{CI}$ : 0.11-0.30) was associated with lower odds for modern contraceptive use (Table 2).

\section{Household and community level factors}

Middle (aOR: 1.52, 95\% CI: 1.28-1.80) and high (aOR: 1.72, 95\% CI: 1.41-2.11) household wealth were positively associated with modern contraceptive in Kenyan women who had experienced high-risk births. Similarly,
Kenyan women from middle (aOR: 1.47, 95\% CI: 1.18 1.54) and high (aOR: 1.66, 95\% CI: 1.27-2.16) household wealth who had experienced under-five mortality had higher odds for using modern contraceptives. Community level factors were largely not associated with contraceptive use in both countries for both women who had experienced high-risk births and under-five mortality, with the exception that, in Zimbabwe, high poverty level (aOR: 0.65, 95\% CI: 0.48-0.89) was associated with lower odds of contraceptive use among women who had high risk births (Table 2).

\section{Multi-level analysis}

After adjusting for individual and community level factors, increasing birth order was positively associated with high-risk births in Kenyan women (aOR: 3.14, 95\% CI: 2.95-3.34), increasing mother's age (aOR: 1.02 , 95\% CI: 1.02-1.04) and middle wealth index quartile (aOR: 1.27 , 95\% CI: 1.06-1.53). Primary (aOR: 0.77, 95\% CI: 0.62-0.96) and secondary education (OR: $0.62,95 \%$ CI: $0.47-0.82$ ) were negatively associated with high-risk births. In Zimbabwe, increasing birth order (aOR: 5.16, 95\% CI: 4.69-5.67) and being previously married (aOR: 1.52, 


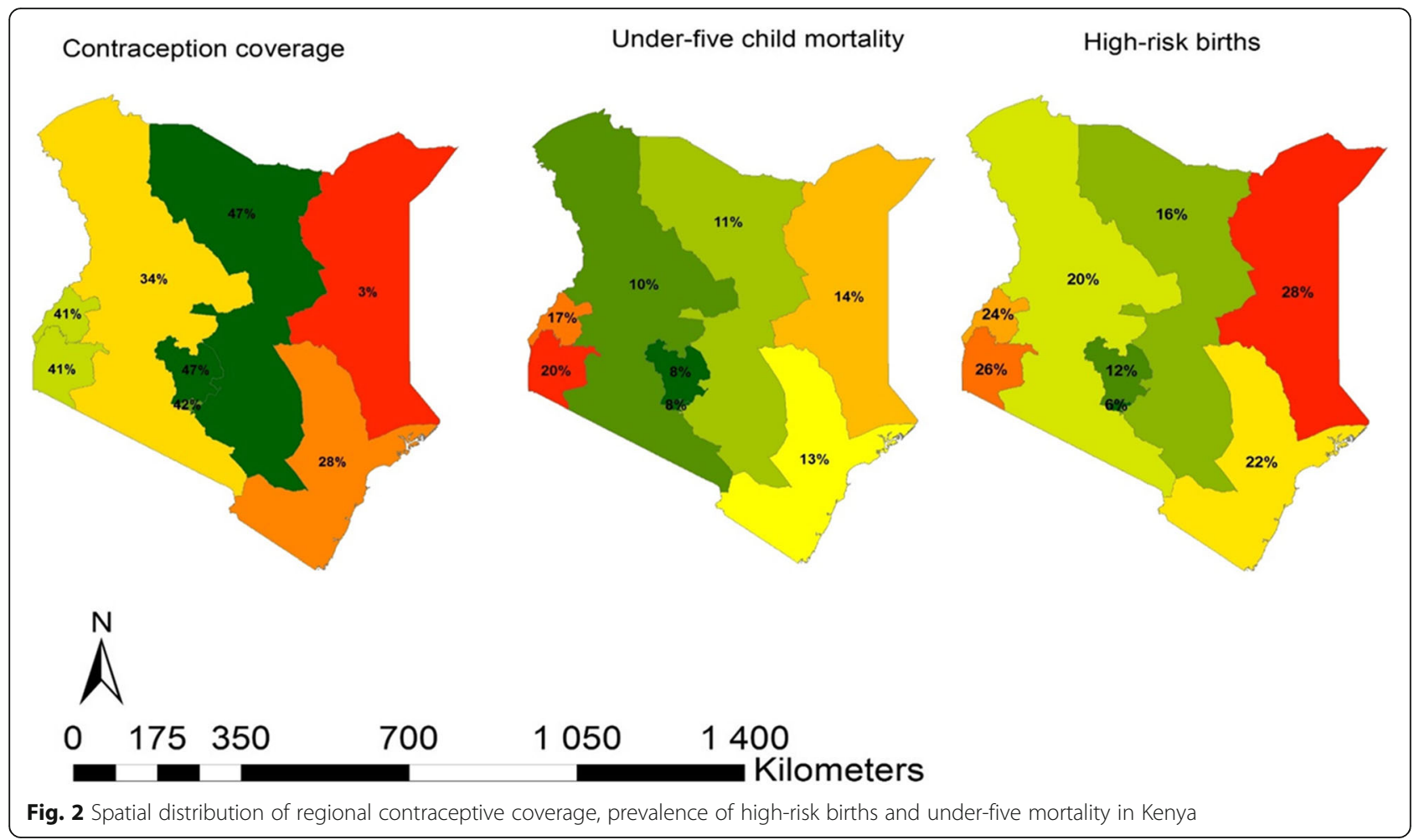

95\% CI: 1.22-1.90) were positively associated with high-risk births. Increasing mother's age (aOR: 0.93, 95\% CI: 0.92-0.94), modern contraceptive use (aOR: 0.79, 95\% CI: 0.68-0.92), primary (aOR: $0.42,95 \%$ CI: 0.24-0.76) and secondary education (aOR: 0.21, 95\% CI: $0.12-0.38$ ) were negatively associated with high-risk births. Community level factors (i.e. place of residence, illiteracy, unemployment and poverty levels) were not significantly associated with high-risk births (Table 3).
Under-five mortality in Kenya were positively associated with a higher birth order (aOR: 1.57, 95\% CI: 1.151.62 ), only primary school education (aOR: $1.30,95 \% \mathrm{CI}$ : $1.07-1.58$ ), and high poverty levels (aOR: 1.33 , 95\% CI: 1.07-1.64). Higher maternal age aOR: $0.99,95 \% \mathrm{CI}$ : 0.98-0.99), using modern contraceptive methods (aOR: 0.79, 95\% CI: $0.70-0.89)$ and living in rural areas (aOR: 0.78, 95\% CI: 0.67-0.91) provided protection against under-five mortality (Table 4). In Zimbabwe, an increasing

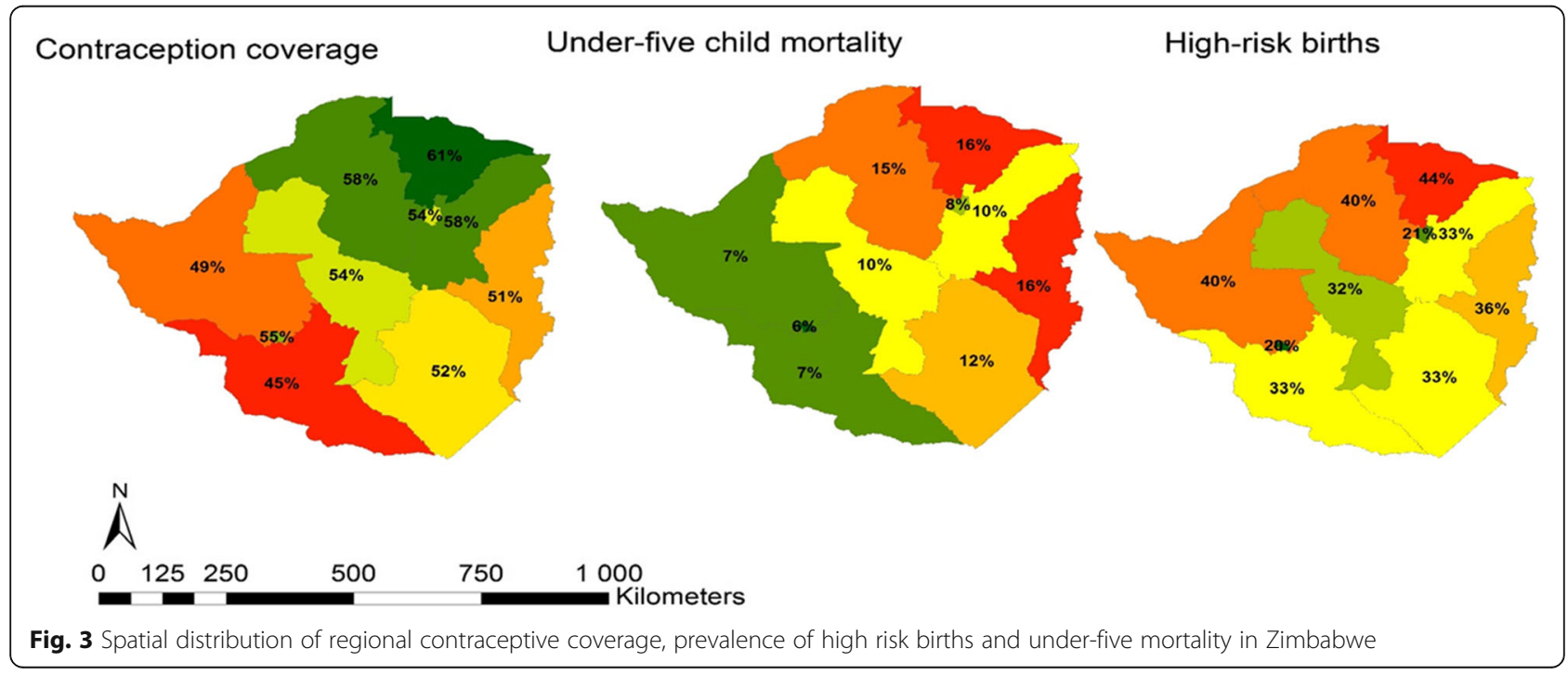




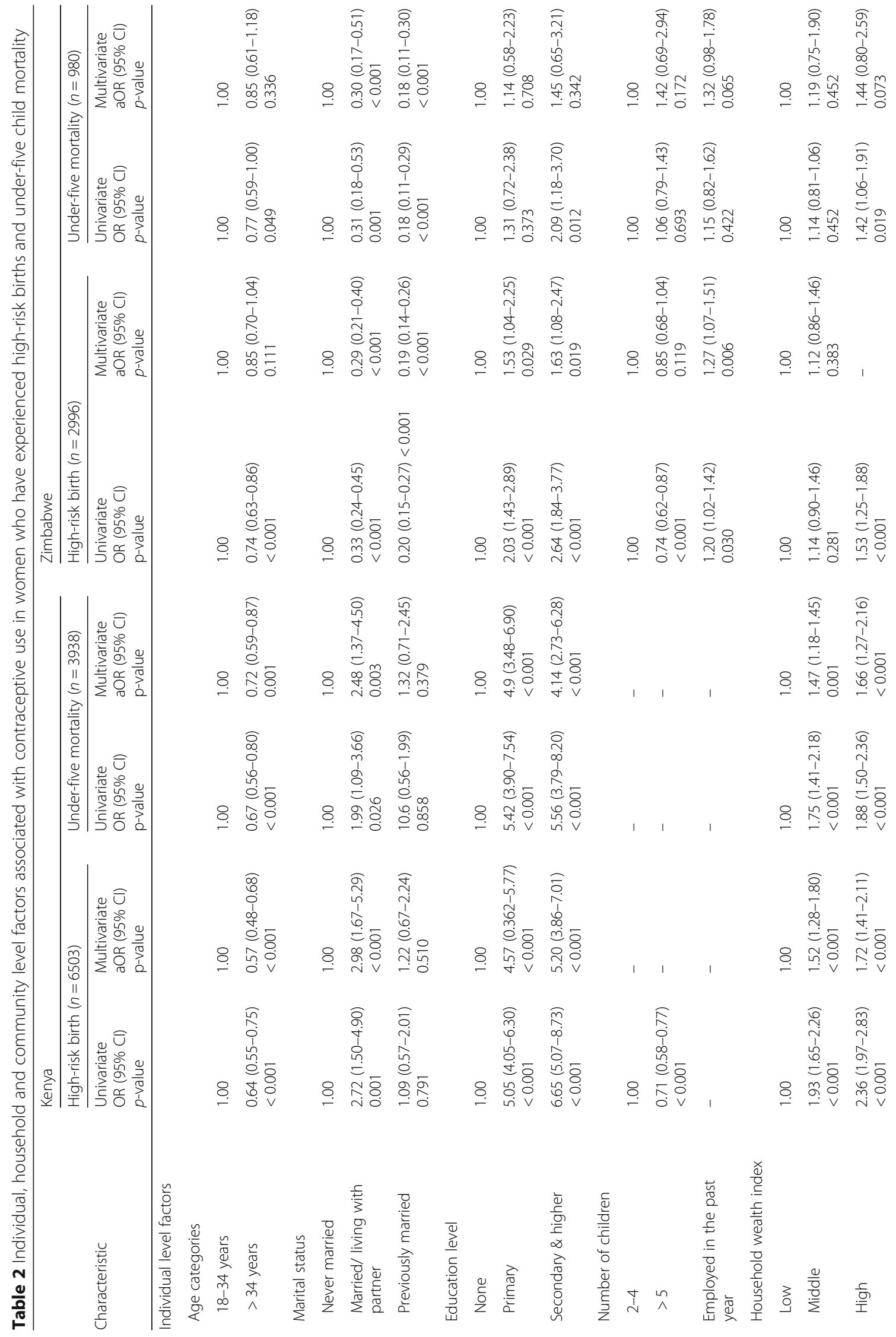




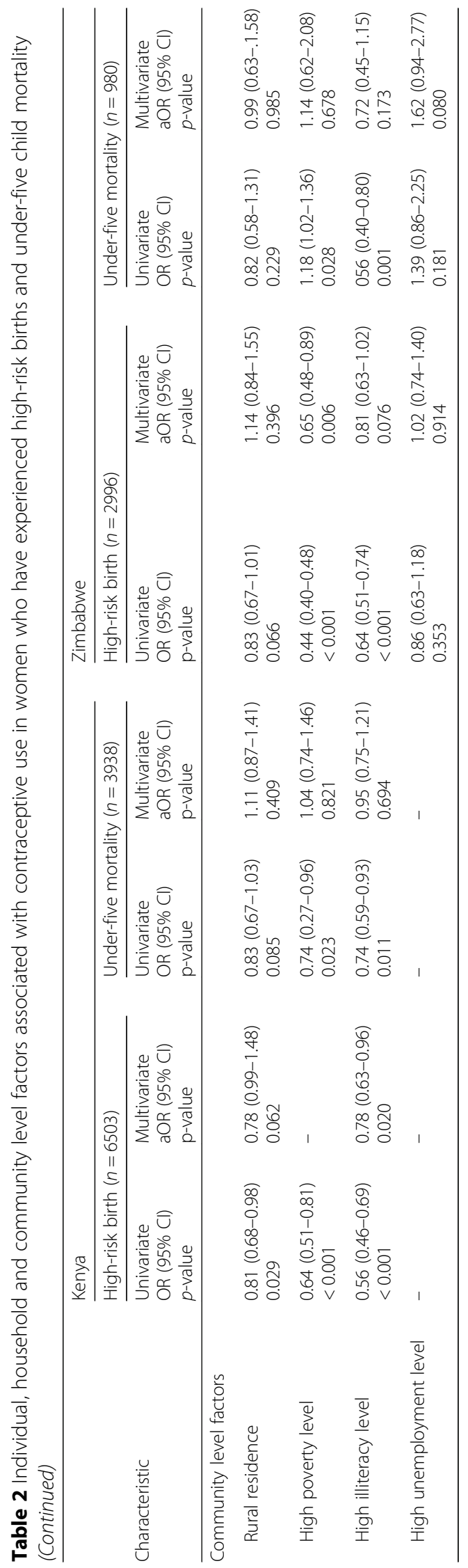




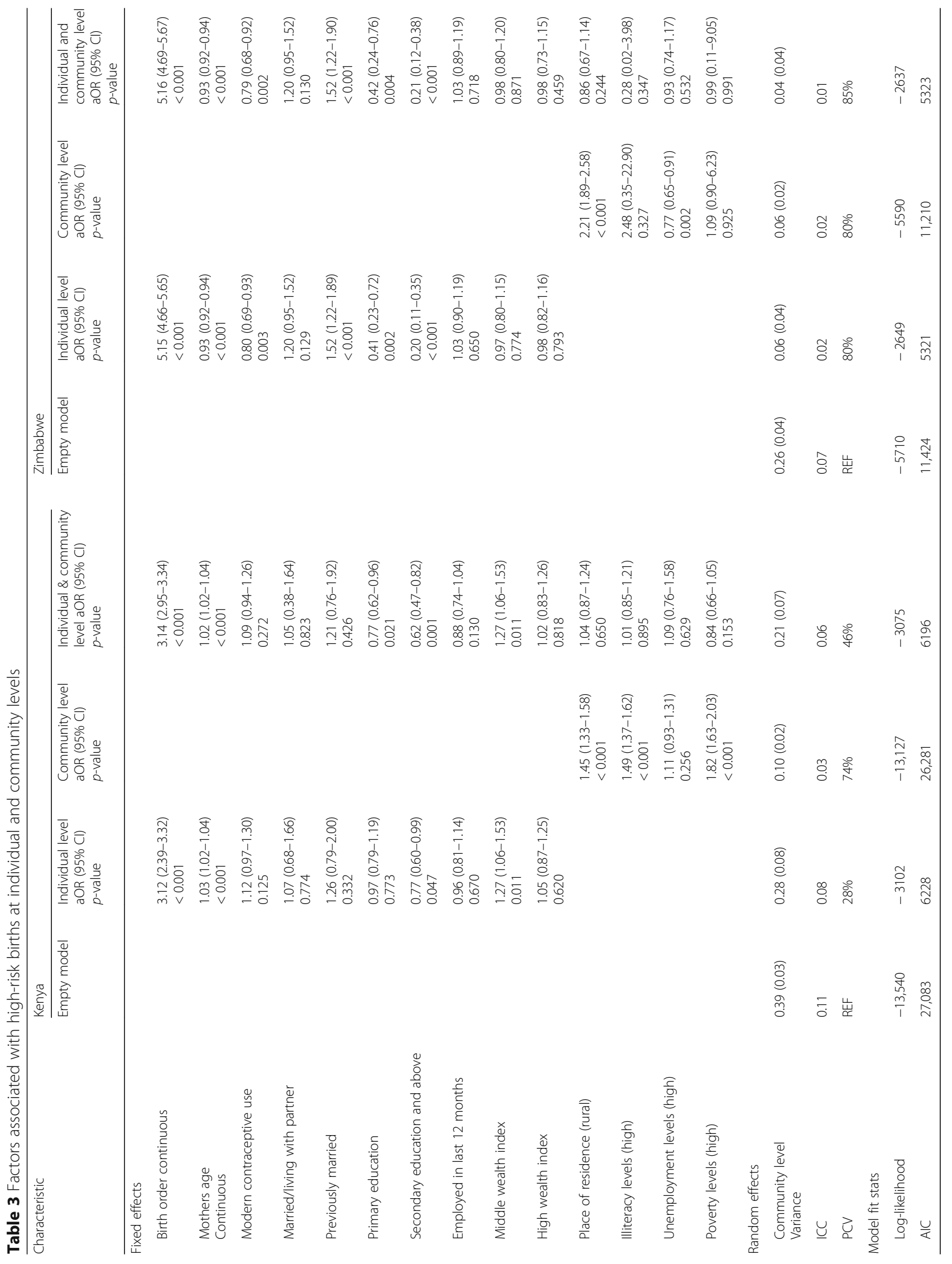




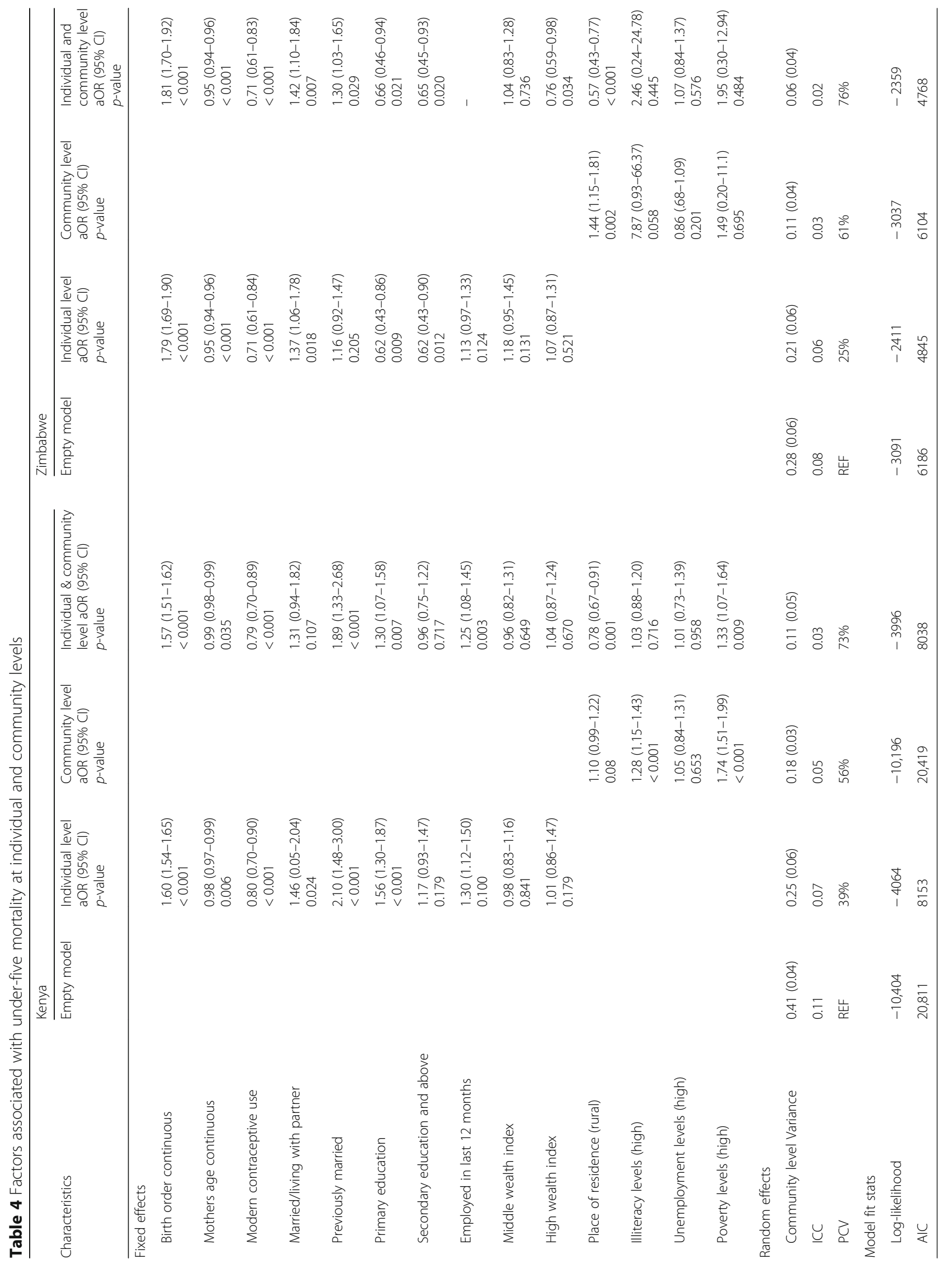


birth order (aOR: 1.81, 95\% CI: 1.70-1.92), living with partner/spouse (aOR: 1.42, 95\% CI: 1.10-1.84) and being previously married (aOR: 1.30, 95\% CI: 1.03-1.1.65) was associated with under-five mortality. Similar to Kenyan women, a protective effect was seen in higher maternal ages (aOR: 0.95, 95\% CI: 0.94-0.96), using modern contraception (aOR: 0.71, 95\% CI: 0.61-0.83) and living in rural areas (aOR: 0.57, 95\% CI: 0.43-0.77). Zimbabwean women with primary (aOR: 0.66, 95\% CI: 0.46-0.94), secondary (aOR: 0.65, 95\% CI: 0.45-0.93) levels of education and a high wealth index (aOR: 0.76, 95\% CI: 0.59-0.98) were less likely to experience under-five child mortality. With the exception of high poverty levels in Kenya (aOR: 0.66, 95\% CI: 0.46-0.94) and place of residence in Zimbabwe (aOR: 0.66, 95\% CI: 0.46-0.94), community level factors were not significantly associated with under-five mortality (Table 4).

\section{Discussion}

In this analysis, we described the spatial distribution of contraceptive coverage, and the prevalence of high-risk births and under-five mortality. We also characterised the factors associated with contraceptive use among women who had experienced high-risk births and under-five mortality in Kenya and Zimbabwe. Contraceptive use prevalence, though less than $50 \%$ in both countries, was lower in Kenya compared to Zimbabwe. The spatial maps, especially in the Kenyan North-eastern region, show an inverse correlation between prevalence of contraceptives use and under-five mortality as well as high risk births. This confirms findings from other published studies [5-7]. However, other regions for example Nyanza, which reported the highest contraceptive rate $(41 \%)$, also reported high rates of high-risk births (26\%) and under-five mortality (20\%) which could be explained by other factors such as high HIV prevalence in this region compared to the rest of the country [23]. In Zimbabwe, the pattern was less clear, for instance Mashonaland central province had the highest prevalence of contraceptive use as well as higher rates of high-risk births and under five mortality. This unexpected finding may be explained by the fact that the province is predominantly rural with limited healthcare services [24]. It is also plausible that the higher contraceptive prevalence is a result of increased efforts by the ministry of health to reduce the unmet need for contraceptive methods in order to address the high-risk births and under five mortality [25, 26].

At an individual level, in both countries, higher levels of education were significantly associated with higher contraceptive usage in women who had experienced high-risk births and under-five mortality. This finding suggests that more educated women were most likely to understand the benefits of family planning using contraceptives as confirmed in a report from a study in Kenya [14]. Kenyan women, who were married or living together with a partner were significantly more likely to use contraception. However, this was the opposite for Zimbabwe, where married women or women living with partners were less likely to use modern contraception. The reason for this difference is unclear, but could be attributed to cultural variations between the two countries. For example, in Zimbabwe reports suggest that using certain contraceptive methods (such as condoms) in marriage could be regarded as evidence that the woman has more than one partner [27].

At a household level, among Kenyan women who had experienced high-risk births in both countries, women from middle or higher household wealth status were at least one and half times more likely to be using contraceptive compared to women from low household incomes. This could be explained by the fact that women who have financial resources have better access to sexual reproductive health services including contraceptives $[28,29]$. It is also supported by our finding that, at community level in Zimbabwe, higher levels of poverty were associated with reduced odds of contraceptive usage.

Multi-level analysis showed that in Zimbabwe, contraceptive use was protective against both high risk births and under-five mortality whilst in Kenya it was only significantly protective against under-five mortality. The effect of contraception use is two-fold as the proportion of high-risk births are reduced and as a result, a decline in child mortality is seen [30]. We also found that maternal education was associated with reduced odds of high risk births and under-five mortality in both countries even though this was more evident in Zimbabwe. It is hypothesised that education protects against high-risk births because young women spend more years in school delaying delivery before 18 years, become more aware of available methods of contraception and tend to be employed, thus have income which can improve their access to health care including contraceptive services $[28,29,31]$. Maternal education provides autonomy in decision making which increases the mother's ability to access health care for children in a household, thus ultimately contributing to the reducing under-five mortality [32]. We also found that high-risk births were positively associated with increasing maternal age and birth-order. This is keeping in line with literature that shows this association $[10,11]$. As confirmed in other studies, a higher maternal age increased child survival in both countries. Younger mothers may lack social and psychological support needed to meet the requirements for childcare [32, 33].

Community level factors, with the exception of high poverty levels in Zimbabwe were not significantly associated with contraceptive use among women who had 
experienced high-risk births or under-five mortality. This finding was in contradiction with a report from Chama-Chiliba and colleagues which highlighted the strong association between community level factors and uptake of antenatal services [15]. The reason for this difference is unclear but could be due the heterogeneity of the group variable for example, the urban areas are generally characterised by better access to sanitation, nutrition and healthcare, but the proliferation of slums in most African cities often mean that a significant proportion of the urban dwellers face overcrowded living conditions, inadequate sanitation and limited access to quality healthcare including access to contraception services.

The main limitation of this study is that it is cross sectional, therefore associations observed do not imply causality. Secondly, the DHS are conducted independently within countries and thus not measured at the same time which limits the contemporaneous crossnational comparisons to some extent. Thirdly, most of the health measures in DHS are based on self-report or proxy report and thus may suffer from recall and social desirability bias. Fourthly, the analysis of factors associated with contraceptive use was limited to women who had experienced high risk births or under-five mortality and this may have introduced bias in the modelling process by excluding other groups such as women with low and medium risk births. Lastly, contraceptive use was current, whilst under-five mortality and high risk births referred to the past 5 years. It may be that if both measures were provided on the same time scales, findings may have differed. Despite these limitations, DHS has several strengths such as: greater population coverage, standardized data collection procedures across countries which are consistent over time and therefore allowing comparability across different settings. Additionally, the multistage probabilistic sampling design of DHS allowed for the multi-level analyses that we conducted to explore community and individual level factors that influenced the outcomes of interest. Furthermore, our analysis provides results from two SSA countries with different settings, thus the findings could be extrapolated to other countries within the region.

\section{Public health and policy implications}

The low contraceptive prevalence rates in both countries suggests that more effort is required to improve uptake of contraception and alleviate unmet needs. The increased contraception coverage would in turn contribute to the reduction of under-five mortality and possibly high-risk births. Improving education and poverty levels of girls and women in the SSA region might help improve contraceptive prevalence rates, decrease high-risk births and under-five mortality in the region.

\section{Conclusions}

There is need to increase contraceptive coverage due to the positive benefits associated with it such as reduction of unplanned pregnancies and high risk-births as well as under-five mortality. Increasing access to education and subsequent economic opportunities for women in sub-Saharan Africa could help achieve multiple improved health benefits.

\section{Additional files}

Additional file 1: Figure S1. Distribution of regional contraceptive coverage, prevalence of high-risk births and under-five mortality in Kenya and Zimbabwe. Show the prevalence of contraceptive use, high-risk births and under-five mortality in Kenya and Zimbabwe stratified by regional/provincial level. (PNG 175 kb)

Additional file 2: Figure S2. Proportion of women using contraception in urban vs. rural regions on Kenya and Zimbabwe. Compares urban and rural contraceptive use in Kenya and Zimbabwe stratified by regional/ provincial level. (PNG $176 \mathrm{~kb}$ )

Additional file 3: Figure S3. Proportion of high-risk births in urban vs. rural regions in Kenya and Zimbabwe. Compares urban and rural high-risk births prevalence in Kenya and Zimbabwe stratified by regional/provincial level. (PNG $189 \mathrm{~kb}$ )

Additional file 4: Figure S4. Proportion of under-five child mortality in urban vs. rural regions of Kenya and Zimbabwe. Compares urban and rural under-five child mortality prevalence in Kenya and Zimbabwe stratified by regional/provincial level. (PNG $180 \mathrm{~kb}$ )

\section{Abbreviations \\ AIC: Akaike Information Criterion; AOR: Adjusted odds ratio; Cl: Confidence Intervals; DHS: Demographic Health Survey; ICC: Intra Community Correlation Coefficient; IQR: Interquartile range; MMR: Maternal mortality ratio; \\ PCV: Proportional Change in Community Variance; PSU: Primary Sampling \\ Unit; SDG: Sustainable Development Goal; SSA: Sub-Saharan Africa; \\ U-5MR: Under-5 mortality rate}

\section{Acknowledgements}

We would like to thank MEASURE DHS for giving us permission to use their data.

\section{Funding}

This research was supported by the Consortium for Advanced Research Training in Africa (CARTA). CARTA is jointly led by the African Population and Health Research Center and the University of the Witwatersrand and funded by the Carnegie Corporation of New York (Grant No--B 8606.R02), Sida (Grant No:54100029), the DELTAS Africa Initiative (Grant No: 107768/Z/15/Z). The DELTAS Africa Initiative is an independent funding scheme of the African Academy of Sciences (AAS)'s Alliance for Accelerating Excellence in Science in Africa (AESA) and supported by the New Partnership for Africa's Development Planning and Coordinating Agency (NEPAD Agency) with funding from the Wellcome Trust (UK) (Grant No: 107768/Z/15/Z) and the UK government. The statements made and views expressed are solely the responsibility of the fellow. The first author is a recipient of the Career Development Fellowship from the EDTCP2 programme supported by the European Union. However, the information or views in this manuscript are those of the authors and EDTCP Association is not responsible for any use that may be made of the information contained in this publication. The first author was supported in part by the National Research Foundation (NRF) of South Africa (Grant Number: 110807), however findings and conclusions expressed in this publication are that of the author and the NRF accepts no liability whatsoever in this regard.

Availability of data and materials

The datasets used for this publication are publicly available at measure DHS website. The link to the website is: http://dhsprogram.com/data/ available-datasets.cfm. 


\section{Authors' contributions}

AC and LC conceptualised and designed the study. LC, EB and KO conducted the data analysis. All authors wrote the manuscript and provided critical review. All authors read and approved the final manuscript.

\section{Ethics approval and consent to participate}

We sought permission and accessed the data from MEASURE DHS database at http://dhsprogram.com/data/available-datasets.cfm. The ORC Macro Inc. has removed all information that could be used to identify the respondents. Hence, no effort was made to identify any household or individual respondent interviewed in the survey. Written informed consent was obtained from all participants before face to face interviews were conducted. Minors provided assent whilst consent was provided by their parents or head of the household.

\section{Consent for publication}

Not applicable

\section{Competing interests}

The authors declare that they have no competing interests.

\section{Publisher's Note}

Springer Nature remains neutral with regard to jurisdictional claims in published maps and institutional affiliations.

\section{Author details}

'Wits RHI, Faculty of Health Sciences, University of the Witwatersrand, 22 Esselen St, Hillbrow, Johannesburg 2001, South Africa. ${ }^{2}$ Perinatal HIV Research Unit, Faculty of Health Sciences, University of the Witwatersrand, Johannesburg, South Africa. ${ }^{3}$ The Aurum Institute, 29 Queens Road, Parktown, Johannesburg 2194, South Africa. ${ }^{4}$ School of Public Health, Faculty of Health Sciences, University of Witwatersrand, Johannesburg, South Africa.

\section{Received: 2 January 2018 Accepted: 15 October 2018} Published online: 24 October 2018

\section{References}

1. Sustainable Development Goals: 17 Goals to Transform Our World. http:// www.un.org/sustainabledevelopment/health/. Accessed 3 Apr 2016.

2. WHO. Trends in maternal mortality: 1990 to 2015 : estimates by WHO, UNICEF, UNFPA, World Bank Group and the United Nations Population Division. Geneva: World Health Organization; 2015.

3. Black RE, Morris SS, Bryce J. Where and why are 10 million children dying every year? Lancet. 2003;361(9376):2226-34.

4. Starbird E, Norton M, Marcus R. Investing in family planning: key to achieving the sustainable development goals. Global Health: Science and Practice. 2016;4(2):191-210

5. Stover J, Ross J. Changes in the distribution of high-risk births associated with changes in contraceptive prevalence. BMC Public Health. 2013;13(3): $1471-2458$.

6. Cramer JC. Social factors and infant mortality: identifying high-risk groups and proximate causes. Demography. 1987;24(3):299-322.

7. Brown W, Ahmed S, Roche N, Sonneveldt E, Darmstadt GL. Impact of family planning programs in reducing high-risk births due to younger and older maternal age, short birth intervals, and high parity. Semin Perinatol. 2015; 39(5):338-44.

8. Maïga A, Hounton S, Amouzou A, Akinyemi A, Shiferaw S, Baya B, Bahan D, Barros AJD, Walker N, Friedman H. Trends and patterns of modern contraceptive use and relationships with high-risk births and child mortality in Burkina Faso. Glob Health Action. 2015;8. https://doi.org/10.3402/gha. v3408.29736.

9. Hubacher D, Mavranezouli I, McGinn E. Unintended pregnancy in subSaharan Africa: magnitude of the problem and potential role of contraceptive implants to alleviate it. Contraception. 2008;78(1):73-8.

10. Fotso JC, Cleland J, Mberu B, Mutua M, Elungata P. Birth spacing and child mortality: an analysis of prospective data from the Nairobi urban health and demographic surveillance system. J Biosoc Sci. 2013;45(6):779-98.

11. Kembo J, Van Ginneken JK. Determinants of infant and child mortality in Zimbabwe: results of multivariate hazard analysis. Demogr Res. 2009;21(13): 367-84.

12. Campbell M, Sahin-Hodoglugil NN, Potts M. Barriers to fertility regulation: a review of the literature. Stud Fam Plan. 2006;37(2):87-98.
13. Konje JC, Ladipo OA. Barriers to uptake and use of modern methods of contraception in developing countries. Int J Gynecol Obstet. 1999;65(3):287-94.

14. Ochako R, Mbondo M, Aloo S, Kaimenyi S, Thompson R, Temmerman M, Kays M. Barriers to modern contraceptive methods uptake among young women in Kenya: a qualitative study. BMC Public Health. 2015;15(118):015-1483.

15. Chama-Chiliba CM, Koch SF. Utilization of focused antenatal care in Zambia: examining individual- and community-level factors using a multilevel analysis. Health Policy Plan. 2015;30(1):78-87.

16. Makate M, Makate C. Prenatal care utilization in Zimbabwe: examining the role of community-level factors. J Epidemiol Global Health. 2017;7(4):255-62.

17. Grepin KA, Bharadwaj P. Maternal education and child mortality in Zimbabwe. J Health Econ. 2015;44:97-117.

18. Kenya National Bureau of Statistics, Ministry of Health/Kenya, National AIDS Control Council/Kenya, Kenya Medical Research Institute, Population NCf, Development/Kenya. Rockville: Kenya Demographic and Health Survey 2014; 2015.

19. Zimbabwe National Statistics Agency, ICF International. Zimbabwe Demographic and Health Survey 2011: Final Report. Rockville: Zimbabwe National Statistics Agency (ZIMSTAT) and ICF International; 2012.

20. Yebyo H, Alemayehu M, Kahsay A. Why do women deliver at home? Multilevel modeling of Ethiopian National Demographic and health survey data. PLoS One. 2015;10(4):e0124718.

21. StataCorp. Stata Statistical Software: Release 14. College Station: StataCorp LP; 2015.

22. ESRI. ArcGIS Desktop: Relaese 10. Redlands: Environmental Systems Research Institute; 2011.

23. NAAC. Kenya Count HIV Profiles: National AIDS Control Council; 2016.

24. Central Statistical Office. Provincial Report Mashonaland Cent. Harare: CSO; 2012.

25. Determinants of unmet need for family planning in Zimbabwe. http://www. znfpc.org.zw/images/pdfs/unmetneed_for_family_planning_2010.pdf. Accessed 15 May 2016

26. The Zimbabwe National Marternal and Neonatal Health Road Map 20072015. http://www.who.int/pmnch/countries/zimbabwe_roadmap_web.pdf. Accessed 21 May 2017

27. Zimbabwe National Statistics Agency, United Nations Fund for Population Activities. Nuptuality and Ferility thematic report. Harare: Population Census Office; 2015.

28. Nanda P. Gender dimensions of user fees: implications for women's utilization of health care. Reprod Health Matters. 2002;10(20):127-34

29. Sharma V, Mohan U, Das V, Awasthi S. Socio demographic determinants and knowledge, attitude, practice: survey of family planning. J Fam Med Prim Care. 2012; (1):43-7.

30. Shiferaw S, Spigt M, Tekie M, Abdullah M, Fantahun M, Dinant G-J. The effects of a locally developed mHealth intervention on delivery and postnatal care utilization; a prospective controlled evaluation among health Centres in Ethiopia. PLoS One. 2016;11(7):e0158600.

31. Nanda G, Schuler SR, Lenzi R. The influence of gender attitudes on contraceptive use in Tanzania: new evidence using husbands' and wives' survey data. J Biosoc Sci. 2013;45(3):331-44.

32. Kanmiki EW, Bawah AA, Agorinya I, Achana FS, Awoonor-Williams JK, Oduro AR, Phillips JF, Akazili J. Socio-economic and demographic determinants of under-five mortality in rural northern Ghana. BMC Int Health Hum Rights. 2014;14:24.

33. Ettarh RR, Kimani J. Determinants of under-five mortality in rural and urban Kenya. Rural Remote Health. 2012;12:1812. 